\title{
Soluble Syndecan-1: Does This Biomarker Address a Seemingly Insoluble Problem in Inflammatory Bowel Disease?
}

\author{
Angela M. Patterson ${ }^{1}$
}

Received: 8 April 2015/Accepted: 10 April 2015/Published online: 25 April 2015

(c) Springer Science+Business Media New York 2015

Syndecans are a major class of cell surface heparan sulfate proteoglycan (HSPG) that help maintain physiological homeostasis. Changes in expression and function of these molecules are associated with and contribute to many pathophysiological conditions including malignancies and inflammation [1, 2]. Syndecans are composed of a core protein comprised of an ectodomain, a transmembrane domain, and a conserved cytoplasmic domain, with three or more structurally diverse heparan sulfate chains. Four individual syndecans are expressed in a highly regulated tissue-, cell-, and development-specific manner [1, 2]. As part of normal cell HSPG turnover, the intact ectodomain is shed from the cell surface, rendering HSPG soluble. Membrane-bound and soluble HSPG can act either as paracrine or autocrine effectors or as negative modulators of cellular signaling events. Syndecan shedding occurs by highly regulated cleavage mechanisms in response to specific developmental and homeostatic signals, but also in response to pathophysiological signals such as inflammatory cytokines and chemokines, as illustrated in Fig. 1 [2, $3]$. Of the four identified syndecans, syndecan-1 is predominantly expressed by plasma cells and mucosal epithelial cells, such as intestinal epithelial cells [3]. Syndecan expression and shedding is highly modulated in inflammation-associated disease states, such as Crohn's disease or ulcerative colitis [1-3].

The intestinal epithelium provides an essential barrier and the first site of immune recognition and response to

Angela M. Patterson

a.patterson@abdn.ac.uk

1 School of Medical Sciences, University of Aberdeen, Life Sciences Innovation Building, Cornhill Road, Aberdeen AB25 2ZS, UK environmental factors, such as antigens and microbes, by limiting their entry and spread from the lumen to the interstitium and then to systemic tissues. Recognition and binding of specific factors by receptors on epithelial cells initiates molecular signaling and induction of local immune responses, followed by production of homeostatic chemokines that regulate leukocyte traffic into and out of the underlying lamina propria. Secreted cytokines modulate the activation and recruitment of lymphocytes and phagocytes to the lamina propria as well as promote cellcell interactions and maintain epithelial barrier integrity [4].

In inflammatory bowel diseases (IBD), these host responses to luminal factors such as bacteria or antigens are defective and poorly regulated, with resultant development of persistent and extensive gut cellular infiltration and inflammation, an increased turnover rate of cell surface molecules and epithelial cells, and disruption to the integrity of the epithelial layer with loss of epithelial cells and distortion of mucosal structure. Currently, IBD is diagnosed by clinical assessment, endoscopic investigation, and laboratory analysis of biochemical markers in blood and tissue obtained by biopsies. These diagnostic factors can readily differentiate between the two main chronic idiopathic IBDs, Crohn's disease (CD) and ulcerative colitis (UC). Yet, the use of these invasive diagnostic procedures can be limiting with regard to monitoring disease activity and assessing disease severity during the treatment, recovery, and remission phases of the disease. There is therefore a need to identify new specific disease biomarkers that do not rely on invasive investigations for diagnosis [5-9].

A characteristic feature of IBD is the loss of intestinal barrier integrity, characterized by major changes in intestinal permeability and the expression of tight junction 
Fig. 1 Syndecan-1 expression within the intestine during homeostasis and inflammation

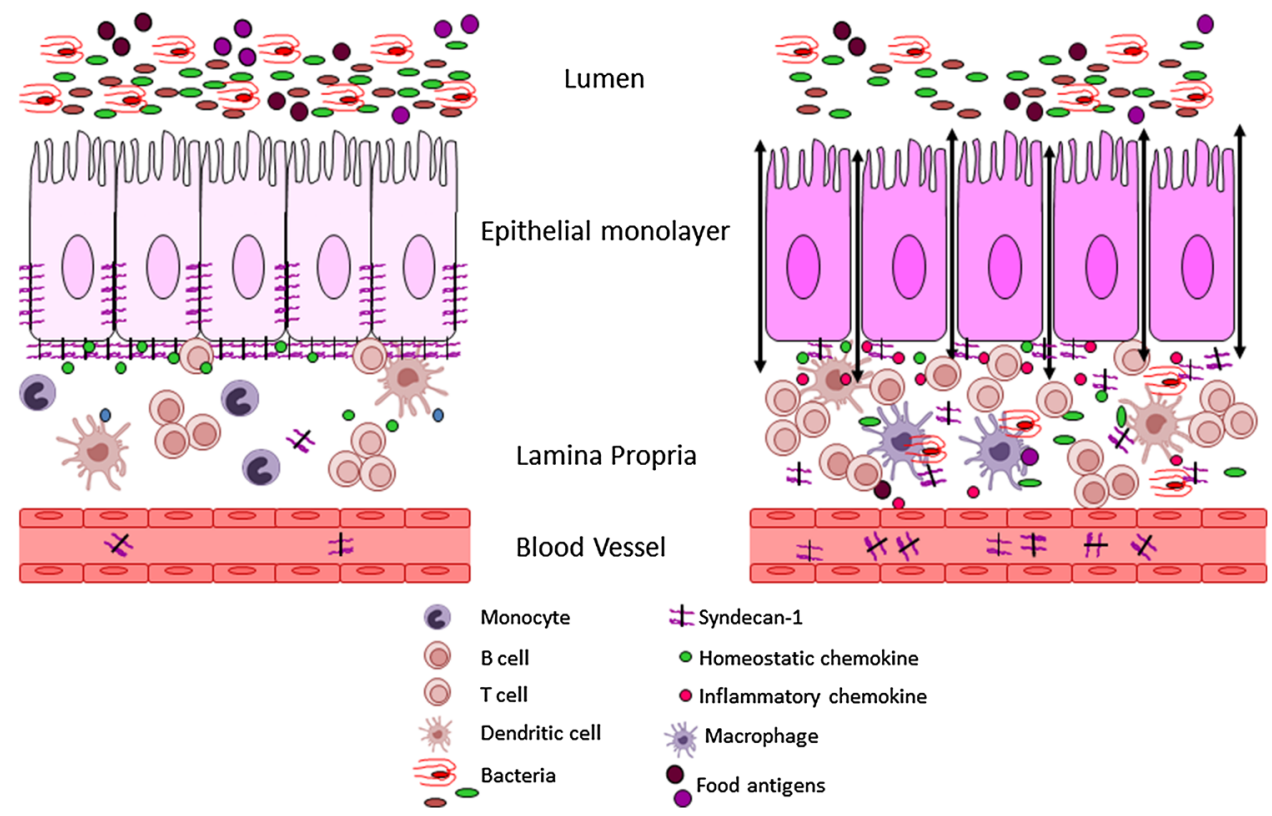

proteins (claudins and occludins) and expression and shedding of syndecan-1 [3]. Intestinal permeability can be assessed by measuring the urinary excretion of carbohydrate probes such as mannitol, lactulose, and sucralose following oral ingestion $[10,11]$. Nonetheless, this is an artificial and indirect measure of barrier function. Thus, monitoring of gut integrity and function continues to be assessed mostly by immunohistochemistry of biopsy specimens [12].

In this context, the aim of the study by Yablecovitch et al. [13] published in this issue of Digestive Diseases and Sciences, was to investigate the correlation between soluble syndecan-1 and IBD disease activity in addition to its use as a diagnostic marker. The authors studied 41 patients with IBD and 16 healthy controls. The cohort of IBD patients was initially divided into two groups, CD and UC, based on existing diagnostic criteria and then further divided into three groups based on the medication regime used. The authors reported a significant difference in soluble concentrations of syndecan-1 between healthy controls and the cohort of IBD patients, which correlated with increased expression of the classical inflammatory marker, C-reactive protein (C-RP), a criterion used in the diagnosis of IBD. Although Yablecovitch et al. suggest that levels of soluble syndecan-1 in patients with CD were higher than in controls, the measurement of soluble syndecan-1 did not have adequate specificity for discriminating between subjects with UC and with controls. The authors, however, did not emphasize the possibility that this biomarker could discriminate between patients with $\mathrm{CD}$ and UC. On further analysis of syndecan-1 levels between the subgroups of IBD patients (treated and untreated active disease patients and patients in remission), no correlation with C-RP levels was observed, likely due to the small number of patients in each category. Another factor that the authors did not take into consideration was the other potential sources of inflammatory processes, all of which are reflected in the measurement of C-RP, such as underlying infections. Therefore, soluble syndecan-1 levels may be more reflective of intestinal inflammation rather than general inflammation.

In conclusion, as the authors have suggested, soluble syndecan-1 levels can be used as a diagnostic marker of inflammatory bowel disease. Predicting the utility of this biomarker for assessing disease activity and progression would have required longitudinally monitoring each subgroup of IBD patients. Recently, identification of biomarkers that correlate with IBD disease activity has been reported by a number of groups [14]. To date, each identified biomarker has potential utility for disease diagnosis and prognostication, although no individual biomarker is sufficiently robust to be used singly for IBD diagnosis or monitoring. In some studies, clinical activity was still viewed as being more reliable than biomarker assessment [15]. It is therefore worth considering selecting a panel of biomarkers for diagnosis and monitoring of disease activity and progression, for example, fecal calprotectin and syndecan-1.

This finding represents a step forward in the quest to diagnose and monitor IBD using noninvasive, readily 
available, reproducible, and easily quantified tests, which will not only improve the management of individual patients, but will also facilitate the study of large populations in the assessment of disease risk factors and responses to novel therapies.

\section{References}

1. Sarrazin S, Lamanna WC, Esko JD. Heparan sulfate proteoglycans. Cold Spring Harb Perspect Biol. 2011;3:a004952.

2. Manon-Jensen T, Itoh Y, Couchman JR. Proteoglycans in health and disease. The multiple roles of syndecan shedding. FEBS $J$. 2010;277:3876-3889.

3. Bode L, Salvestrini C, Park PW, et al. Heparan sulphate and syndecan-1 are essential in maintaining murine and human intestinal epithelial barrier function. J Clin Invest. 2008;118: 229-238.

4. Peterson LW, Artis D. Intestinal epithelial cells: regulators of barrier function and immune homeostatis. Nat Rev Immunol. 2014; 14:141-153.

5. Lewis JD. The utility of biomarkers in the diagnosis and therapy of inflammatory bowel disease. Gastroenterology. 2011;140; $1817-1826$.

6. Iskandar HN, Ciorba MA. Biomarkers in inflammatory bowel disease: current practices and recent advances. Transl Res. 2012; 159:313-325.
7. Vaiopoulou A, Gazouli M, Theodoropoulos G, et al. Current advantages in the application of proteomics in inflammatory bowel disease. Dig Dis Sci. 2012;57:2755-2764.

8. Bennike T, Birkelund S, Stensballe A, et al. Biomarkers in inflammatory bowel diseases: current status and proteomics identification strategies. World J Gastroenterol. 2014;20:3231-3244.

9. Cioffi M, Rosa AD, Serao R, et al. Laboratory markers in ulcerative colitis: current insights and future advances. World $J$ Gastrointest Pathophysiol. 2015;6:13-22.

10. Vogelsang H. Do changes in intestinal permeability predict disease relapse in Crohn's disease? Inflamm Bowel Dis. 2008;14(Suppl 2): S162-S163.

11. Teshima CW, Dieleman LA, Meddings JB. Abnormal intestinal permeability in Crohn's disease pathogenesis. Ann N Y Acad Sci. 2012;1258:159-165.

12. Lee SH. Intestinal permeability regulation by tight junction: implication on inflammatory bowel diseases. Intest Res. 2015;13: 11-18.

13. Yablecovitch D, Stein A, Shabat-Simon M. Soluble syndecan-1 levels are elevated in patients with inflammatory bowel disease. Dig Dis Sci. 2015 (Epub ahead of print). doi:10.1007/s10620015-3589-9.

14. Pardi DS, Sandborn WJ. Predicting relapse in patients with inflammatory bowel disease: what is the role of biomarkers. Gut. 2005;54:321-322.

15. Schoepfer AM, Vavricka S, Zahnd-Straumann $\mathrm{N}$, et al. Monitoring inflammatory bowel disease activity: clinical activity is judged to be more relevant than endoscopic severity or biomarkers. J Crohns Colitis. 2012;6:412-418. 